\title{
Endoscopic plastic stent therapy for bile leaks following total vs subtotal cholecystectomy
}

\section{다 (요우}

\author{
Authors \\ Patrick Yachimski, Jordan K. Orr, Anthony Gamboa
}

Institution

Division of Gastroenterology, Hepatology \& Nutrition, Vanderbilt University Medical Center, Nashville Tennessee, United States

submitted 30.6.2020

accepted after revision 5.10 .2020

\section{Bibliography}

Endoscopy International Open 2020; 08: E1895-E1899

DOI 10.1055/a-1300-1319

ISSN 2364-3722

(C) 2020. The Author(s).

This is an open access article published by Thieme under the terms of the Creative Commons Attribution-NonDerivative-NonCommercial License, permitting copying and reproduction so long as the original work is given appropriate credit. Contents may not be used for commecial purposes, or adapted, remixed, transformed or built upon. (https://creativecommons.org/licenses/by-nc-nd/4.0/)

\section{Corresponding author}

Patrick S. Yachimski, MD MPH FASGE AGAF, Vanderbilt University Medical Center, 1660 The Vanderbilt Clinic, Nashville, TN 37232-5280, United States

Fax: +1-6153437174

Patrick.yachimski@vumc.org

\section{ABSTRACT}

Background and study aims Plastic biliary stents are standard therapy for treatment of post-cholecystectomy bile leaks. An increasing proportion of patients now under- go subtotal cholecystectomy and are at perceived risk for high-grade bile leak. Data are limited regarding the optimal endoscopic therapy following subtotal cholecystectomy. The aim of this study was to compare outcomes of endoscopic plastic stent therapy for treatment of bile leak following total vs subtotal cholecystectomy.

Patients and methods A retrospective cohort of patients with bile leak following cholecystectomy and treated with biliary stent was identified from an institutional database. Primary outcome was defined as cholangiographic resolution of leak at follow-up endoscopic retrograde cholangiopancreatography (ERCP).

Results Sixty-one subjects met study inclusion criteria, 27 following total cholecystectomy and 34 following subtotal cholecystectomy. A single plastic biliary stent was placed in $87 \%$ of subjects (53/61), while a fully covered self-expanding metal stent (FCSEMS) was placed in $13 \%(8 / 61)$. Leak resolution was evident at first follow-up ERCP in $96 \%$ of subjects (26/27) who had undergone total cholecystectomy and $91 \%$ of subjects (31/34) who had undergone subtotal cholecystectomy $(P=0.25)$. Among subjects who had received a plastic stent at index ERCP, leak resolution was evident at first follow-up ERCP in $96 \%(23 / 24)$ of those who had undergone total cholecystectomy and $90 \%$ (26/ $29)$ of those who had undergone subtotal cholecystectomy $(P=0.62)$.

Conclusions High rates of leak resolution can be achieved with placement of a single plastic biliary stent for treatment of post-cholecystectomy bile leaks, including after subtotal cholecystectomy.

\section{Introduction}

Cholecystectomy is one of the most commonly performed surgeries. Cholecystectomy is typically approached in a minimally invasive laparoscopic fashion. The incidence of bile leak following laparoscopic cholecystectomy is estimated to be approximately $1 \%$ [1]. While a significant percentage of bile leaks resolve spontaneously and without clinical sequelae in the early post-perative period, persistent bile leaks typically require therapeutic intervention with endoscopic retrograde cholangiopancreatography (ERCP). Among patients with post-chole- cystectomy bile leak who undergo ERCP, the cystic duct stump is the most frequently identified site of leak [1,2].

The goal of endoscopic management in patients requiring ERCP for post-cholecystectomy bile leak without bile duct injury is to decrease the pressure gradient at the level of the papilla, thereby creating a high flow pathway to siphon bile flow into the duodenum and away from the site of leak to permit spontaneous leak healing and closure. This can be achieved by placement of a biliary endoprosthesis, with or without biliary sphincterotomy, or conceivably in some instances by biliary sphincterotomy alone [2,3]. Plastic biliary stents are first-line 
therapy for this indication given their efficacy, ease of placement and subsequent removal, and low cost. Typical practice at our institution is to place the shortest plastic stent available in order to minimize resistance to transpapillary flow, as based on principles of fluid dynamics resistance to laminar flow increases with increasing stent length.

In some patients undergoing cholecystectomy factors such as severe gallbladder inflammation or limited anatomic exposure may prevent safe completion of a standard laparoscopic cholecystectomy. In such cases, surgical options include conversion to an open surgical approach and/or conversion to a subtotal cholecystectomy. Options for subtotal cholecystectomy include a fenestrated technique in which the neck of residual gallbladder is left open without attempt at ligation or closure, or a reconstituted technique in which closure of the remaining gallbladder is attempted resulting in an intact gallbladder lumen [4]. The prevalence of bile leaks following subtotal cholecystectomy may be as high as $18 \%$ [5], and patients who have undergone subtotal cholecystectomy are more likely to require post-operative ERCP compared with patients who have undergone standard/total cholecystectomy [6].

In our clinical experience, and although stent selection is ultimately at the discretion of the endoscopist, a consultative request to perform ERCP for treatment of bile leak following subtotal cholecystectomy is often accompanied by a request from the surgical team to place a fully covered self expanding metal stent (FCSEMS) rather than a plastic stent as therapeutic management. Given the perceived high-grade nature of the leak, the premise is that a FCSEMS is necessary to "seal off" the cystic duct and prevent leakage from the residual gallbladder. While FCSEMS have expanded indications for treatment of benign biliary disease including benign bile duct strictures [7], and while use of FCSEMS has been reported for successful management of refractory bile duct leaks [8,9], to our knowledge controlled or comparative data have not been reported to either question the utility of plastic stents or support the use of FCSEMS as firstline management for bile duct leaks following subtotal cholecystectomy.

The aim of this study was to compare outcomes of endoscopic plastic stent therapy for post-cholecystectomy bile leak in patients undergoing standard (total) vs subtotal cholecystectomy. The a priori hypothesis was that there would be no difference in leak resolution rates following plastic stent placement in patients undergoing standard vs subtotal cholecystectomy.

\section{Patients and methods}

This was a single-center retrospective study, with approval for study conduct granted by the Vanderbilt University Medical Center Institutional Review Board.

Patients who had undergone ERCP for treatment of postcholecystectomy bile leak between 2009 and 2020 were identified from an endoscopy database consisting of the cases of two interventional endoscopists (PY and AG). Study inclusion criteria were strictly restricted to patients with surgically unaltered foregut anatomy who had undergone both cholecystectomy and ERCP at our institution. Patients who had undergone cholecystectomy at an outside institution and who were then transferred to our institution for management of bile leak, for whom full operative details of cholecystectomy were not available for review, were excluded. Patients identified at ERCP to have leaks originating from the gallbladder fossa/duct of Luschka were excluded, as were patients found at ERCP to have common bile duct or right posterior sectoral duct injury. Patients who underwent ERCP for bile leak following blunt or penetrating abdominal trauma, bile leak following partial hepatectomy, or anastomotic bile leak following orthotopic liver transplantation were also excluded. Patients lost to follow-up or otherwise without follow-up ERCP at our institution to confirm leak resolution were excluded.

Demographic and clinical data were extracted from the electronic medical record. Operative notes from cholecystectomy and ERCP were manually reviewed to identify relevant technical and procedural details. A high-grade bile leak was defined as cholangiographic evidence of bile leak without requirement for balloon occlusion cholangiography; conversely, a low-grade bile leak was defined as cholangiographic evidence of bile leak elicited only by balloon occlusion cholangiogram.

The primary outcome of interest was resolution of bile leak on repeat interval ERCP. Standard protocol at our institution is to schedule repeat elective ERCP at 6 to 8 weeks following placement of a stent for a bile leak, unless warranted sooner for reintervention if there is clinical suspicion for refractory leak. Additional outcomes of interest included need for additional endoscopic, radiologic, or surgical intervention for refractory bile leak. In cases requiring additional endoscopic intervention, procedural details were noted. Adverse events (AEs) associated with ERCP were recorded including pancreatitis, hemorrhage, or perforation, with pancreatitis severity graded according to standard criteria proposed by Cotton et al [10].

Extracted data were stored in de-identified fashion in REDCap (Research Electronic Data Capture) housed at Vanderbilt University. REDCap is a secure, web-based application that provides an intuitive user interface that streamlines project development and improves data entry through real-time validation rules (with automated data type and range checks). REDCap also provides easy data manipulation (with audit trails for reporting, monitoring and querying patient records) and an automated export mechanism to common statistical packages [11].

Statistical analysis was performed using IBM SPSS Statistics 26 (Armonk, New York, United States). Univariate testing of significance for categorical values was performed using Chi square or Fisher's exact testing. Univariate testing of significance for continuous variables was performed using one-way analysis of variance (ANOVA). A two-sided $P<0.05$ was accepted as the threshold for statistical significance.

\section{Results}

\section{Demographics}

A total of 61 subjects fulfilled study inclusion criteria. Of subjects, $53 \%$ (32/61) were male and mean age was 53.1 years. The most common indication for cholecystectomy was acute 
- Table 1 Cohort demographic and cholecystectomy details.

\begin{tabular}{|l|c|}
\hline & $\mathbf{N}=\mathbf{6 1}$ total \\
\hline Age & 53.1 years \\
\hline Male sex & $32(53 \%)$ \\
\hline Current smoker & $7(12 \%)$ \\
\hline Preoperative ERCP performed & $9(15 \%)$ \\
\hline Indication for cholecystectomy & \\
\hline - Acute cholecystitis & $44(72 \%)$ \\
\hline - Gallstone pancreatitis without cholecystitis & $8(13 \%)$ \\
\hline - Symptomatic cholelithiasis & $7(12 \%)$ \\
\hline - Other & $2(3 \%)$ \\
\hline Surgical approach & \\
\hline - Laparoscopic & $4474 \%)$ \\
\hline - Laparoscopic converted to open & $14(23 \%)$ \\
\hline - Open & $2(3 \%)$ \\
\hline ERCP, endoscopic retrograde cholangiopancreatography. & \\
\hline
\end{tabular}

cholecystitis (72\%) and the operative approach was laparoscopic in the majority of cases (74\%). Of the patients, $44 \%$ (27/61) had undergone total/standard cholecystectomy while $56 \%$ (34/61) had undergone subtotal cholecystectomy. Patients who underwent subtotal cholecystectomy were more likely to be male (65\% vs $37 \%, P=0.03$ ) and more likely to be older (58.6 years vs 46.2 years, $P<0.01$ ) than patients who underwent total cholecystectomy. Full demographic details and details of cholecystectomy are listed in $>$ Table 1.

\section{Technical aspects of ERCP}

Mean time to ERCP following cholecystectomy was 5.2 days. High-grade bile leak was evident in $51 \%$ of cases (31/61). Biliary sphincterotomy was performed in $67 \%$ of cases $(41 / 61)$. A single plastic stent was placed as the index endoprosthesis in $87 \%$ of cases (53/61), while FCSEMS was placed in $13 \%$ of cases ( $8 /$ 61 ). Full details of index endoscopic intervention are listed in - Table 2 .

\section{Adverse events of ERCP}

Identified AEs associated with ERCP were limited to two cases of pancreatitis, one of which was mild and one of which was moderate in severity. There were no instances of sphincterotomyinduced hemorrhage requiring either immediate or delayed endoscopic intervention, and no instances of duodenal or retroperitoneal perforation.

\section{Clinical outcomes of ERCP and stent therapy}

There was no difference in the proportion of patients receiving plastic stents vs FCSEMS, and no difference in the proportion of patients receiving biliary sphincterotomy when comparing the total and subtotal cholecystectomy groups. Difference in mean time to ERCP following total cholecystectomy (6.6 days)
- Table 2 ERCP details.

\begin{tabular}{|c|c|}
\hline & $\mathrm{N}=61$ total \\
\hline High-grade bile leak identified & $31(51 \%)$ \\
\hline Choledocholithiasis identified at ERCP & $10(16 \%)$ \\
\hline Biliary sphincterotomy performed & $41(67 \%)$ \\
\hline Plastic biliary stent placed & $53(87 \%)$ \\
\hline FCSEMS placed & $8(13 \%)$ \\
\hline \multicolumn{2}{|l|}{ Plastic biliary stent diameter: } \\
\hline . $10 \mathrm{~F}$ & $4992 \%)$ \\
\hline - $8.5 \mathrm{~F}$ & $4(8 \%)$ \\
\hline \multicolumn{2}{|l|}{ Plastic biliary stent length: } \\
\hline - $5 \mathrm{~cm}$ & $3464 \%)$ \\
\hline . $7 \mathrm{~cm}$ & $8(15 \%)$ \\
\hline . $8 \mathrm{~cm}$ & $6(11 \%)$ \\
\hline - $10 \mathrm{~m}$ & $4(8 \%)$ \\
\hline . $12 \mathrm{~cm}$ & $1(2 \%)$ \\
\hline \multicolumn{2}{|l|}{ FCSEMS diameter: } \\
\hline . $10 \mathrm{~mm}$ & $4(50 \%)$ \\
\hline . $8 \mathrm{~mm}$ & $4(50 \%)$ \\
\hline \multicolumn{2}{|l|}{ FCSEMS length: } \\
\hline . $4 \mathrm{~cm}$ & $1(13 \%)$ \\
\hline - $6 \mathrm{~cm}$ & $5(63 \%)$ \\
\hline - $8 \mathrm{~cm}$ & $2(25 \%)$ \\
\hline $\begin{array}{l}\text { ERCP, endoscopy retrograde cholangiopanc } \\
\text { ered self-expanding metal stent }\end{array}$ & SEMS, fully cov- \\
\hline
\end{tabular}

vs subtotal cholecystectomy (4.1 days) did not meet the threshold for statistical significance $(P=0.10)$. Comparison of demographic and treatment details in the total vs subtotal cholecystectomy groups is depicted in $>$ Table 3 .

Overall leak resolution rate following index ERCP with stent placement was $96 \%$ (26/27) following total cholecystectomy and $91 \%$ (31/34) following subtotal cholecystectomy $(P=$ 0.25). Mean time to repeat ERCP for stent removal was 53.2 days following total cholecystectomy and 54.9 days following subtotal cholecystectomy. Excluding patients treated with FCSEMS, $96 \%$ of patients $(23 / 24)$ treated with plastic stents following total cholecystectomy achieved complete leak resolution without requirement for reintervention compared to $90 \%$ of subjects (26/29) treated with plastic stents following subtotal cholecystectomy $(P=0.62)$.

Of the four patients with evidence of persistent leak on follow-up ERCP, all had been treated with plastic stents at the index ERCP (one following total cholecystectomy, three following subtotal cholecystectomy). At repeat ERCP documenting persistent leak, all were treated with extended duration stent therapy (mean 134 days, range 53 -309). One patient also required 
- Table3 Demographic and ERCP comparison of total vs subtotal cholecystectomy groups.

\begin{tabular}{|c|c|c|c|}
\hline & Total & Subtotal & $P$ value \\
\hline & $N=27$ & $N=34$ & \\
\hline Age & 46.2 years & 58.6 years & $<0.01$ \\
\hline Male & $10(37 \%)$ & $22(65 \%)$ & 0.03 \\
\hline High-grade bile leak identified & $11(41 \%)$ & $20(59 \%)$ & 0.16 \\
\hline Choledocholithiasis identified at ERCP & $5(19 \%)$ & $5(15 \%)$ & 0.74 \\
\hline Biliary sphincterotomy performed & $19(70 \%)$ & $22(65 \%)$ & 0.64 \\
\hline Plastic biliary stent placed & $24(89 \%)$ & $29(85 \%)$ & 1.0 \\
\hline FCSEMS placed & $3(11 \%)$ & $5(15 \%)$ & \\
\hline Time from cholecystectomy to ERCP & 6.6 days & 4.1 days & 0.10 \\
\hline
\end{tabular}

interventional radiology intervention, consisting of percutaneous drainage of a biloma, for refractory bile leak following index ERCP with stent placement. An additional patient treated with FCSEMS at index ERCP following subtotal cholecystectomy and who underwent stent removal with documentation of leak resolution at follow-up ERCP, presented in delayed fashion with recurrent biloma and underwent repeat ERCP with replacement of a plastic biliary stent as well as radiologic intervention with percutaneous fibrin glue injection. No patients required surgical reintervention for refractory bile leak. All achieved ultimate leak resolution.

\section{Discussion}

Subtotal cholecystectomy has emerged as a viable option for surgical management of gallbladder disease in technically complex cases, with data suggesting that the practice of subtotal cholecystectomy has increased considerably over the past decades. A study from the National Inpatient Sample reported that the rate of laparoscopic subtotal cholecystectomy increased from $0.12 \%$ of all cholecystectomies in 2003 to $0.28 \%$ in 2014 , and the rate of open subtotal cholecystectomy increased from $0.10 \%$ to $0.52 \%$ within the same timeframe. Factors associated with likelihood of subtotal cholecystectomy in this study included male sex, Asian American ethnicity, surgery performed in a teaching hospital, and surgery performed in a rural location [12]. ERCP with biliary stent placement is first-line therapy for treatment of bile leaks following cholecystectomy. Endoscopic intervention offers high rates of durable leak resolution in minimally invasive fashion and with low rates of morbidity. Yet scant data are available reporting outcomes of endoscopic therapy for bile leaks specifically following subtotal cholecystectomy, which was a focus of this study. Given the reported high prevalence of bile leaks following subtotal cholecystectomy [5], continued increase in the practice of subtotal cholecystectomy would have major implications on post-cholecystectomy ERCP volume and mandates a need for high-quality data to guide optimal endoscopic management for this patient population.
This study reports high rates of leak resolution (91\%) with biliary stent placement following subtotal cholecystectomy, including a $90 \%$ resolution rate with a single plastic stent as used in the majority of patients in this cohort. Although not evaluated in this study, placement of multiple plastic biliary stents for treatment of high-grade leaks [13] could also be an alternative to single plastic stents or FCSEMS.

This study has clear limitations. The size of the cohort was smaller than anticipated, as due to the stringent inclusion and exclusion criteria the majority of patients who underwent ERCP for treatment of bile leak during the study time period were excluded from analysis. Due to the single-center, retrospective nature of the study as well as the strict exclusion criteria, the modest size of the cohort may result in a type II error, failing to detect a true difference in leak resolution rates following total vs subtotal cholecystectomy. For instance, if the true refractory leak rate is $4 \%$ for plastic stent therapy following total cholecystectomy and $10 \%$ following subtotal cholecystectomy, a study would require 378 subjects in each group to detect this difference with $90 \%$ power at an alpha level of 0.05 .

As an additional limitation, the size of the cohort precluded meaningful multiple variable analysis to both control for potential confounders and identify factors associated with refractory leak. A larger cohort would be necessary to identify independent associations between refractory leak rates and specific technical aspects of cholecystectomy and/or technical aspects of ERCP (plastic stent vs FCSEMS, performance of biliary sphicterotomy, etc).

Furthermore, the objective criteria used to adjudicate outcomes and categorize data do not eliminate the fact that there is some heterogeneity in endoscopic techniques within the cohort. Performance of biliary sphincterotomy was not standardized, and stent selection was at the discretion of the treating endoscopist. These endoscopic variables likely reflect the contribution of subjective judgment of the endoscopist, perhaps influenced by worry about the size, location, or characteristics of a given leak and affecting treatment decisions at the time of the procedure, yet not granular in the operative report at the 
time of data extraction. With respect to cholecystectomy operative reports, variability in the detail of these notes limited consistent assessment of whether subtotal cholecystectomy was of the fenestrated or reconstituted type [4], which could conceivably impact the nature of the post-operative leak and response to endoscopic therapy.

\section{Conclusions}

In summary, in this retrospective study the majority of patients undergoing treatment for bile leak following either total or subtotal cholecystectomy were treated with a single plastic biliary stent. This study demonstrated no difference in the rate of successful leak resolution with plastic biliary stent placement following total vs subtotal cholecystectomy. These data support the use of plastic stents as first-line therapy for bile leaks following either total or subtotal cholecystectomy.

\section{Acknowledgment}

REDCap is supported by UL1 TR000445 from NCATS/NIH.

\section{Competing interests}

Dr. Yachimski is a consultant for Boston Scientific.

\section{References}

[1] Barkun AN, Rezieg M, Mehta SN. McGill Gallstone Treatment Group. . et al. Postcholecystectomy bile leaks in the laparoscopic era: risk factors, presentation, and management. Gastrointest Endosc 1997; 45: 277-282

[2] Sandha GS, Bourke M], Haber GB et al. Endoscopic therapy for bile leak based on a new classification: results in 207 patients. Gastrointest Endosc 2004; 60: 567-574
[3] Kaffes A], Hourigan L, De Luca N et al. Impact of endoscopic intervention in 100 consecutive patients with suspected postcholecystectomy bile leak. Gastrointest Endosc 2005; 61: 269-275

[4] Strasberg SM, Pucci M], Brunt LM et al. Subtotal cholecystectomy "fenestrating" vs "reconstituted" subtypes and the prevention of bile duct injury: definition of the optimal procedure in difficult operative conditions. J Am Coll Surg 2016; 222: 89-96

[5] Elshaer M, Gravante G, Thomas K et al. Subtotal cholecystectomy for "difficult gallbladders": systematic review and meta-analysis. JAMA Surg 2015; 150: 159-168

[6] Lidsky ME, Speicher PJ, Ezekian B et al. Subtotal cholecystectomy for the hostile gallbladder: failure to control the cystic duct results in significant morbidity. HPB (Oxford) 2017; 19: 547-556

[7] Coté GA, Slivka A, Tarnasky P et al. Effect of covered metallic stents compared with plastic stents on benign biliary stricture resolution: a randomized clinical trial. JAMA 2016; 315: 1250-1257

[8] Kahaleh M, Sundaram V, Condron SL et al. Temporary placement of covered self-expandable metallic stents in patients with biliary leak: midterm evaluation of a pilot study. Gastrointest Endosc 2007; 66: 52-59

[9] Canena ], Liberato M, Meireles L et al. A non-randomized study in consecutive patients with postcholecystectomy refractory bile leaks who were managed endoscopically with the use of multiple plastic stents or fully-covered self-expandable metal stents (with videos). Gastrointest Endosc 2015; 82: 70-78

[10] Cotton PB, Lehman G, Vennes J et al. Endoscopic sphincterotomy complications and their management: an attempt at consensus. Gastrointest Endosc 1991; 37: 383-393

[11] Harris PA, Taylor R, Thielke R et al. Research electronic data capture (REDCap)-a metadata-driven methodology and workflow process for providing translational research informatics support. J Biomed Inform 2009; 42: 377-381

[12] Sabour AF, Matsushima K, Love BE et al. Nationwide trends in use of subtotal cholecystectomy for acute cholecystitis. Surgery 2020; 167 : 569-574

[13] Canena J, Horta D, Coimbra J et al. Outcomes of endoscopic management of primary and refrctory postcholecystectomy bile leaks in a multicentre review of 178 patients. BMC Gastroenterol 2015; 15: 105 